\title{
Coagulation in Liver Surgery: An Observational Haemostatic Profile and Thromboelastography (TEG) Study (CLOTS).
}

June Oo (MBBS, FRACS) ${ }^{1}$, Megan Allen (BMBS (Hons), FANZCA) 2,3, Benjamin PT Loveday (PhD, FRACS $)^{1,4,5}$, Nora Lee (MBBS, FRACP) ${ }^{6}$, Brett Knowles (MBChB, FRACS) 1,5, Bernhard Riedel (MD, PhD) 2,3, Kate Burbury (FRACP/FRCPA, DPhil) ${ }^{6,8}$, Benjamin Thomson (MBBS, FRACS) ${ }^{1,7}$

1. Department of Surgical Oncology, Peter MacCallum Cancer Centre, Melbourne, Victoria, Australia

2. Department of Anaesthesia, Perioperative and Pain Medicine, Peter MacCallum Cancer Centre, Melbourne, Victoria, Australia

3. Centre for Integrated Critical Care, Melbourne Medical School, University of Melbourne, Victoria, Australia

4. Department of Surgery, University of Auckland, Auckland, New Zealand

5. Department of Surgery, Royal Melbourne Hospital, Melbourne, Victoria, Australia

6. Department of Hematology, Peter MacCallum Cancer Centre and Royal Melbourne Hospital, Melbourne, Victoria, Australia

This is the author manuscript accepted for publication and has undergone full peer review but has not been through the copyediting, typesetting, pagination and proofreading process, which may lead to differences between this version and the Version of Record. Please cite this article as doi: 10.1111/ans.15912

This article is protected by copyright. All rights reserved. 
7. Department of Surgery, University of Melbourne, Melbourne, Victoria, Australia

8. Sir Peter MacCallum Cancer Centre, University of Melbourne, Melbourne, Victoria, Australia

\section{Corresponding Author Contact Details}

Associate Professor Benjamin Thomson

Department of Surgical Oncology

Peter MacCallum Cancer Centre

305 Grattan Street

Melbourne VIC 3000

Australia

Ben.thomson@petermac.org

Manuscript category: Original article

Funding: Charitable Feilam Foundation, Perth, Western Australia for haematological testing

Conflict of interest: None

This article is protected by copyright. All rights reserved. 


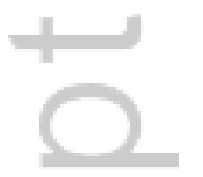




\begin{abstract}
Introduction. International Normalised Ratio (INR) is used as a marker of the haemostatic status following liver resection. However, the impact of liver resection on haemostasis is complex and beyond what can be measured by INR. This study aimed to prospectively assess haemostatic profile following liver resection and determine if INR measurement can safely guide postoperative thromboprophylaxis.

Methods. In this prospective cohort study, patients undergoing liver resection had coagulation parameters (INR, PT, aPTT, fibrinogen, d-Dimer, von-Willebrand factor antigen, procoagulant activity of phospholipids, clotting factors II, VII, VIIIc, IX and X) and thromboelastogram parameters assessed perioperatively. Clinical follow-up assessed for thromboembolism and haemorrhage.

Results. In the forty-one patients included, INR was significantly $(\mathrm{p}<0.0001)$ elevated postoperatively, and INR >1.5 was observed in 7/41 (17.1\%) on POD1 and 1/41 (2.4\%) patients on POD3, respectively. Factor VII levels showed transient reduction but other factors, especially factor II and X, remained within normal range following liver resection. Thromboelastogram parameters remained normal or supranormal for all patients at all time points. One incident of post hepatectomy haemorrhage occurred, despite a normal coagulation profile. Two patients suffered late pulmonary embolic episodes.
\end{abstract}

Conclusion. Post liver resection haemostasis is complex and poorly reflected by INR, which should not guide initiation of chemical thromboprophylaxis in the immediate postoperative period.

This article is protected by copyright. All rights reserved. 
Key Words: liver resection, coagulation, INR, clotting factors, TEG

\section{Introduction}

Liver resection may alter postoperative laboratory coagulation profile, especially the international normalized ratio (INR). However, the thrombo-haemorrhagic risk of INR derangement is unclear (1). Altered hepatic synthetic function and metabolism following liver resection may cause both haemorrhage and thromboembolism (2, 3). Patients undergoing liver resection often have additional risk factors that both increase and decrease coagulation potential including advanced age, malignancy, prolonged surgery, and blood loss (4).

Currently, INR is often used clinically to guide timing of postoperative thromboprophylaxis (5). When an INR is elevated, chemical thromboprophylaxis may be withheld due to the potential risk of bleeding $(6,7)$. However, there is evidence of transient hypercoagulability after liver resection, even in the presence of an elevated INR $(8,9)$. This may be because INR provides a limited measure of vitamin $\mathrm{K}$ dependent clotting factors rather than in vivo coagulation overall. Therefore, INR lacks predictive capacity of thrombo-haemorrhagic risk in the complex environment following liver resection. Consequently, withholding thromboprophylaxis post liver 
resection based solely on INR thresholds may be detrimental, as thromboembolism remains a preventable cause of morbidity and mortality after liver surgery $(2,10,11)$.

We aimed to prospectively quantify the perioperative haemostatic profile, using conventional coagulation tests in parallel with clotting factor levels and a global haemostatic assay (thromboelastography), and to determine the relationship between an abnormal INR and these parameters. Secondary outcomes in our study cohort were the incidence of early inpatient and late (up to 12 months post surgery) bleeding and thromboembolic events (incidental and symptomatic) and the incidence of postoperative liver failure. 


\section{Methods}

This prospective observational cohort study was approved by the Peter MacCallum Cancer Centre Ethics Committee (approval number 13_52L, 11/07/2013) and written informed patient consent was obtained.

\section{Study population}

All patients 18 years and above scheduled for liver resection for any pathology at Peter MacCallum Cancer Centre (Melbourne, Australia) between August 2013 and June 2015 were eligible for inclusion. Exclusion criteria included requirement for bridging therapeutic anticoagulation, not proceeding with liver resection and requirement for multiple liver resections in the study period. The treatment strategy, including extent of liver resection and selection of thromboprophylaxis was clinician directed.

\section{Surgery}

Surgery was performed using either an open or laparoscopic approach under low central venous pressure (CVP target $<5 \mathrm{cmH}_{2} \mathrm{O}$ ). Liver transection was undertaken with Cavitron ultrasonic surgical aspiratory (CUSA, Integra LifeSciences, New Jersey, USA) for open surgery and Ligasure (Medtronic, Minnesota, USA) or Thunderbeat (Olympus, Tokyo, Japan) for laparoscopic surgery. Major resection was defined as removal or three or more segments, as per the Brisbane Consensus (12).

This article is protected by copyright. All rights reserved. 
Assessment of coagulation profile

Blood samples for coagulation and thrombogenic biomarkers were taken at induction of anaesthesia (baseline), post-operative day (POD) 0 (end of surgery), 1 and 3 using a Sarstedt (Nümbrecht, Germany) monovette blood collection system into EDTA and trisodium citrate solution at $0.109 \mathrm{~mol} / \mathrm{L}$. Baseline and POD0 bloods were taken prior to enoxaparin administration, whilst POD1 and 3 blood collection were not standardized as enoxaparin has negligible impact on activated partial thromboplastin time (aPTT) and its derivatives.

Full blood count parameters (EDTA) were measured on the Cell-Dyn Sapphire automated haematology analyser (Abbott, Abbott Park, Illinois, USA). Citrate tube was used for thromboelastrography (TEG) parameters (TEG-R, TEG-K, TEG-Angle, TEG-MA) using Hemonetics TEG-5000 Thromboelastograph Hemostasis Analyzer (Baintree, MA, USA). The remaining citrated samples were centrifuged at 2,000xg for 15 minutes to provide platelet poor plasma. One aliquot was used for APTT, fibrinogen, PT and INR derivation (Stago-STA automated platforms, coagulometer specific ISI value 1.31, Stago ANZ, Doncaster, Victoria, Australia), whilst two aliquots were double spun and frozen (-80 degrees Celsius) for assessment of clotting factors (II, VII, VIIIc, IX and X), von willebrand factor antigen (vWF-Ag), and procoagulant activity of phospholipid (PPL) (STA-procoag-PPL, Asnières sur Seine Cedex, France).

This article is protected by copyright. All rights reserved. 


\section{Clinical data}

Clinical data were collected prospectively during the inpatient stay, clinic appointments, and supplemented from patient records. This included demographic details, American Society of Anesthesiologists Physical Status (ASA) classification, body mass index, medical comorbidities, type of surgical procedure, complications, and pathology and radiology results. Post-hepatectomy hemorrhage (PHH) and liver failure (PHLF) as defined by International Study Group of Liver Surgery(13) together with thrombo-embolic events (venous and arterial) were clinically monitored. Follow up data was collected at 4-6 weeks and 12 months post operatively.

\section{Statistical analysis}

Patient characteristics, laboratory measurements and clinical outcomes were summarised with descriptive statistics. Continuous variables were reported as median (IQR range). Categorical data were expressed as counts (n) and percentages (\%). Wilcoxon signed-rank test was used to compare median factor levels and other laboratory measurements at POD 0, 1 and 3 to baseline (Prism GraphPad v8.0.2, San Diego, C.A., USA). Coagulation parameters against INR were assessed with Fisher's exact test with significance set at $<0.05$. INR of 1.5 was selected as the cutoff between normal and abnormal INR as bleeding risk associated with major surgery is considered minimal at a level $<1.5(14)$.

\section{Results}

This article is protected by copyright. All rights reserved. 
Forty-eight patients were enrolled in the study with seven subsequently excluded. Six patients did not proceed to liver resection due to intraoperative findings of unresectable hepatic lesions $(n=4)$, presence of extra hepatic metastasis $(n=1)$, or intraoperative findings of extra-hepatic biliary disease only, such that planned liver resection was not required $(n=1)$. One patient was excluded as two separate liver resections were performed during the study period. Three patients were excluded from POD 3 analysis as two were discharged prior to assessment and one patient had insufficient blood collected for analysis.

\section{Baseline characteristics}

The median age was 61 (range: 49 - 73.5) years. Twenty-three patients (56.1\%) were male. The most common pathology was colorectal liver metastasis $(n=29,70.7 \%)$ (Table 1). Open surgery $(n=33,80.4 \%)$ was more commonly performed than laparoscopic procedures (n=8, 19.5\%). Major resections were performed in 14/41 (34.1\%) patients with only two (4.8\%) requiring portal vein embolization. The median operative time was 200 (range: 155 - 272) minutes, and the median estimated blood loss (EBL) was 150ml (range: 100 - 250). Pringle manoeuvre was performed in majority of patients $(\mathrm{n}=35,85.3 \%)$ for a median duration of 30 minutes (IQR: 17 37).

Baseline thrombotic/bleeding risk

This article is protected by copyright. All rights reserved. 
No patients in the cohort had known coagulopathy or thrombophilia. Four patients (9.7\%) were anti-coagulated preoperatively; $3 / 4$ (75\%) with enoxaparin for prior thrombotic event (withheld 24 hours pre-operatively) and 1/4 (25\%) with rivaroxaban for cardiac history (withheld 72 hours pre-operatively). Two patients (4.8\%) were taking aspirin. This was continued peri-operatively in one patient and withheld in the other (surgeon choice). Non-prescription medicines that could affect coagulation were not taken peri-operatively.

\section{Fluid management and thromboprophylaxis}

Peri-operative intravenous fluid therapy administered in the study cohort, are shown in Table 1. Haemoglobin and haematocrit levels are illustrated in Figure S1. No patients received intraoperative transfusion of blood products (except albumin). Enoxaparin was administered intra-operatively in 2/41 patients (4.9\%), in 22/41 (53.6\%) on POD 0, in 38/41 (92.7\%) on POD1 and in all patients who were still in hospital on POD 3. Of the 19 patients in whom enoxaparin was withheld on POD 0, concerns about bleeding were cited in 10; no information was available about the remaining nine patients or the three patients who didn't receive enoxaparin on POD 1 (INR $<1.7$, no clinical evidence of bleeding). The treating team were not blinded to the INR results and all patients with INR > 1.5 received enoxaparin.

\section{Coagulation profile}

This article is protected by copyright. All rights reserved. 
All patients had aPTT, INR, fibrinogen and d-Dimer levels within the normal range at baseline and at end of surgery. Temporal results for aPTT, INR, fibrinogen and dDimer are shown in Figure S2. These demonstrated an increase from baseline in INR $(\mathrm{p}<0.0001)$ and aPTT $(\mathrm{p}<0.0001)$ on POD 1. INR remained elevated on POD 3 ( $<<$ 0.0001) and aPTT continued to rise $(\mathrm{p}<0.0001)$. Fibrinogen rose on POD $1(\mathrm{p}=$ 0.002) and POD $3(\mathrm{p}<0.0001)$.

All factor levels remained within or above the normal reference range at all time points except for four patients who had unexplained low pre-operative factor levels FII 12\% (n=1), FVII 10\% (n=1), FIX 13\% (n=1), FVIII 3\% $(n=1)$. Other parameters, including INR and TEG were normal in these patients. Changes in Factors II, VII, VIIIc, IX and X levels over time are shown in Figure S3. Overall, there was no change from baseline for Factors II, VIIIc, IX and X. However, Factor VII (the factor with the shortest half-life) (15) decreased from baseline on POD1 ( $<<0.0001$ ), but levels began to increase on POD3. Levels of vWF-Ag and PPL showed a marginal significant change from baseline but remained within the normal range at all time points (Figure S3).

All median TEG parameters remained within normal reference range at all time points, and no values differed significantly from baseline (Figure S4).

This article is protected by copyright. All rights reserved. 
The relationships between INR and all the other coagulation profile parameters are shown in Figures 1, 2, S5. On POD 1, seven patients had an INR >1.5. In all seven patients, levels of Factors II, VIII and IX remained above 50\% (within normal range). Factor VII and X reached nadirs of $23 \%$ and $40 \%$, respectively. On POD 3, one patient had an INR >1.5. In this patient, clotting factor levels were as follows: II 50\%, VII 36\%, IX 96\%, and X 44\%.

\section{Clinical outcomes}

There were no instances of PHLF. One instance of grade A PHH was recorded where two units of blood were transfused for a decline in haemoglobin level ( $\mathrm{Hb} 71 \mathrm{~g} / \mathrm{L}$, baseline $106 \mathrm{~g} / \mathrm{L}$ ) on POD 3 post sub-segmental resection. EBL for this patient was 450ml and INR was 1.1 and 1.0 on POD 1 and POD 3, respectively. A second patient received two units packed red blood cells on POD 4 ( $\mathrm{Hb} 84 \mathrm{~g} / \mathrm{L}$, baseline $88 \mathrm{~g} / \mathrm{L}$ ) post right posterior sectionectomy with EBL of $200 \mathrm{~mL}$, and INR 1.8 and 1.0 on POD 1 and 3, respectively. Enoxaparin was not withheld for either patient.

Majority of patients (27/41) were discharged with extended pharmacological thromboprophylaxis as per institutional protocol(16), and therapeutic anticoagulation was recommenced for the four patients within 48 hours post operatively. There were no index thromboembolic events up to 30 days post-operation. Two patients who underwent minor liver resection for colorectal metastases suffered pulmonary embolism 3 and 6 months postoperatively, respectively. Prophylactic enoxaparin was 
commenced POD 0 for both patients, one received extended prophylaxis for 28 days. At the 12-month assessment, ten patients (24.4\%) had disease recurrence and two patients had died.

\section{Discussion}

This prospective cohort study defined a comprehensive coagulation profile following liver resection in 41 patients from a single institution. We demonstrated that there was a transient rise in INR from baseline, which was associated with a minor and transient decrease in Factor VII levels only - the likely explanation for the increased INR. Other factor levels remained within normal range with no significant change from baseline. Importantly, TEG parameters remained within normal limits, even in the presence of an elevated INR. Only $17 \%$ of patients developed an INR $>1.5$; one of these patients required a blood transfusion for low haemoglobin but did not meet the criteria for PHH. Another patient met the criteria for Grade A PHH on POD 3, although their coagulation profile was within normal limits. There were no early thromboembolic events, although two patients developed pulmonary emboli at 3 months and 6 months after surgery. No radiological cancer recurrence was noted in either patient.

Haemostasis is a highly conserved and complex dynamic process that maintains the integrity of the vascular circuitry and its surrounding microenvironment (15). Its roles include maintenance of patency of blood vessels and fluidity of the blood, prevention 
or termination of blood loss from disrupted blood vessels and the removal of the fibrin network when no longer required (17). It also forms a part of the biological continuum required for post-surgical inflammation and repair and depends on the coordination of many cellular and protein components, including cytokines. The liver remains an important synthetic and metabolic source for many of these components(18). The effect of liver resection on this complex system is not well understood. On the one hand, patients may be procoagulant due to underlying malignancy, prolonged general anaesthesia and release of procoagulant factors (e.g. vWF and factor VIII) from the cut surfaces of the liver $(19,20)$. On the other hand, the effect may be potentially hypocoagulable, predominantly due to a decrease in synthesis of activator coagulation factors by the remnant liver, raising concerns about postoperative bleeding $(3,4)$.

Traditionally, INR has been used as a guide to the coagulation status in this setting, and in particular, the decision around application of chemical thromboprophylaxis which is typically withheld if INR is elevated. INR is a conventional screening laboratory test, for which Factor VII is the predominant rate-limiting factor (21). Along with other conventional screening tests, such as APTT, it has been traditionally used to identify patients at risk of bleeding. However these assays were developed primarily to monitor anticoagulant activity, specifically when using vitamin $\mathrm{K}$ antagonists such as warfarin. They were not developed to predict the risk of bleeding in a peri-operative setting and lack the specificity and sensitivity for this purpose (22). 
Furthermore, these tests only measure part of the process of thrombin generation and do not incorporate other aspects of in vivo haemostasis (23). For example, elevated vWF levels, reduced protein C and anti thrombin levels observed post liver resection suggestive of a pro-thrombotic state is not reflected by INR measurement (24-26).

Due to the lack of dynamic assessment of clot formation and stabilization through the standard coagulation assays, viscoelastic assays have been used to determine functional coagulation profile following liver resection. These studies have demonstrated either a normo-coagulopathic or transient hyper-coagulopathic state post liver surgery despite an elevated INR $(8,24)$. Clotting factor levels have also been studied in the peri-operative setting. One recent study measured factor levels in 71 patients on preoperative warfarin with a median INR of 2.24. The median factor levels remained within the wide band required for adequate haemostasis (27\%, 45\%, $50 \%$ and $13 \%$ for Factors II, VII, IX and X respectively)(21). Therefore, it would appear INR is an inadequate laboratory assessment of the complex coagulation status of a patient post liver resection as it is predominantly dependent on factor VII levels.

Thromboembolism is a serious potential complication following liver resection $(3,9)$ with a reported overall incidence of clinical venous thromboembolism of $3.6 \%$ in a study of 419 major resections with a higher incidence in patients without thromboprophylaxis (6.3\% vs. 2.2\%, $\mathrm{p}=0.030)(10)$. Enoxaparin predominantly reduces active factor II and X levels. Factor II and X levels were adequately 
maintained in our cohort, and therefore prophylactic enoxaparin is safe to administer post liver surgery, even in the presence of an elevated INR. A more liberal approach to the administration of pharmacological thromboprophylaxis has the potential to reduce the risk of thromboembolism and its associated morbidity and mortality.

Study limitations included a small cohort with only fourteen major resections. Therefore the impact of major resection on haemostasis could not be comprehensively studied. Most of our cohort had normal baseline liver function therefore the impact of resection on haemostasis in those with pre-existing liver derangement was not assessed. Furthermore, the INR derangements observed were mild - the majority of patients had an INR of 1.5 or less, with only one patient with an INR of 1.8. Consequently, this study is limited in the conclusions that can be drawn for patients with a significantly prolonged elevation in their INR. However, in our cohort normal coagulation function was demonstrated on TEG analysis despite elevated INR levels. The decision to administer chemical prophylactic may be better guided by patient and surgical risk of TE, with the addition of functional coagulation testing (e.g. TEG) in patients with a persistently elevated INR on POD 3.

The overall haemostatic potential for patients post liver resection is complex and cannot be reduced to a single laboratory measurement such as INR. INR does increase after liver resection, although still usually remains below 1.5. Even when INR is $>1.5$, the ability to form a stable clot on thromboelastography remains intact. Therefore, 
INR should not be used to guide decisions about thromboprophylaxis after liver resection, particularly in the immediate postoperative period.

\section{References}

1. Siu J, McCall J, Connor S. Systematic review of pathophysiological changes following hepatic resection. HPB. 2014;16(5):407-21.

2. Baltatzis M, Low R, Stathakis P, Sheen AJ, Siriwardena AK, Jamdar S. Efficacy and safety of pharmacological venous thromboembolism prophylaxis following liver resection: a systematic review and meta-analysis. HPB. 2017;19(4):289-96.

3. Russell MC. Complications following hepatectomy. Surgical Oncology Clinics. 2015;24(1):73-96.

4. Wrighton LJ, O’Bosky KR, Namm JP, Senthil M. Postoperative management after hepatic resection. Journal of Gastrointestinal Oncology. 2012;3(1):41-7. 5. Ramsay MAE, Trotter JF. The INR is only one side of the coagulation cascade: time to watch the clot. Anaesthesia. 2016;71(6):613-7.

6. Nathan H, Weiss MJ, Soff GA, Stempel M, DeMatteo RP, Allen PJ, et al. Pharmacologic Prophylaxis, Postoperative INR, and Risk of Venous

Thromboembolism after Hepatectomy. Journal of Gastrointestinal Surgery. 2014;18(2):295-303.

7. Weiss MJ, Kim Y, Ejaz A, Spolverato G, Haut ER, Hirose K, et al. Venous thromboembolic prophylaxis after a hepatic resection: patterns of care among liver surgeons. HPB. 2014;16(10):892-8. 
8. Barton JS, Riha GM, Differding JA, Underwood SJ, Curren JL, Sheppard BC, et al. Coagulopathy after a liver resection: is it over diagnosed and over treated? HPB. 2013;15(11):865-71.

9. Melloul E, Dondéro F, Vilgrain V, Raptis DA, Paugam-Burtz C, Belghiti J. Pulmonary embolism after elective liver resection: A prospective analysis of risk factors. Journal of Hepatology. 2012;57(6):1268-75.

10. Reddy SK, Turley RS, Barbas AS, Steel JL, Tsung A, Marsh JW, et al. Postoperative pharmacologic thromboprophylaxis after major hepatectomy. Journal of Gastrointestinal Surgery. 2011;15(9):1602-10.

11. Tzeng C-WD, Katz MHG, Fleming JB, Pisters PWT, Lee JE, Abdalla EK, et al. Risk of venous thromboembolism outweighs post-hepatectomy bleeding complications: analysis of 5651 National Surgical Quality Improvement Program patients. HPB. 2012;14(8):506-13.

12. Strasberg SM, Belghiti J, Clavien PA, Gadzijev E, Garden JO, Lau WY, et al. The Brisbane 2000 Terminology of Liver Anatomy and Resections. HPB. 2000;2(3):333-9.

13. Birgin E, Tesfazgi W, Knoth M, Wilhelm TJ, Post S, Ruckert F. Evaluation of the New ISGLS Definitions of Typical Posthepatectomy Complications. Scand J Surg. 2019;108(2):130-6.

14. Tran HA, Chunilal SD, Harper PL, Tran H, Wood EM, Gallus AS. An update of consensus guidelines for warfarin reversal. Medical Journal of Australia. 2013;198(4):198-9.

15. Palta S, Saroa R, Palta A. Overview of the coagulation system. Indian journal of anaesthesia. 2014;58(5):515-23.

16. Surgical Thrombo-embolism Prevention (STEP) protocol: patients with cancer [Internet]. Peter MacCallum Cancer Center. 2016 [cited 23 Nov 2019]. Available from:

http://wwwlegacy/clinicianscorner/testsite/Resources/anaesthesia guidelines. htm - AnaesthesiaGuidelines.

17. Gale AJ. Continuing education course \#2: current understanding of hemostasis. Toxicologic pathology. 2011;39(1):273-80.

18. Roberts HR, Cederbaum AI. The liver and blood coagulation: physiology and pathology. Gastroenterology. 1972;63(2):297-320.

19. Cerutti E, Stratta C, Romagnoli R, Schellino MM, Skurzak S, Rizzetto M, et al. Thromboelastogram monitoring in the perioperative period of hepatectomy for adult living liver donation. Liver Transpl. 2004;10(2):289-94.

20. Ejaz A, Spolverato G, Kim Y, Lucas DL, Lau B, Weiss M, et al. Defining incidence and risk factors of venous thromboemolism after hepatectomy. Journal of gastrointestinal surgery : official journal of the Society for Surgery of the Alimentary Tract. 2014;18(6):1116-24.

21. Burbury KL, Milner A, Snooks B, Jupe D, Westerman DA. Short-term warfarin reversal for elective surgery--using low-dose intravenous vitamin $\mathrm{K}$ : safe, reliable and convenient*. Br J Haematol. 2011;154(5):626-34. 
22. Liontos L, Fralick M, Longmore A, Hicks LK, Sholzberg M. Bleeding Risk Using INR/aPTT Pre-Surgery: Systemtic Review (BRUISR) (BRUISR). Blood. 2017;130(Suppl 1):4654-.

23. Favaloro EJ, Lippi G. Coagulation update: what's new in hemostasis testing? Thromb Res. 2011;127 Suppl 2:S13-6.

24. Mallett SV, Sugavanam A, Krzanicki DA, Patel S, Broomhead RH, Davidson $\mathrm{BR}$, et al. Alterations in coagulation following major liver resection. Anaesthesia. 2016;71(6):657-68.

25. Potze W, Alkozai EM, Adelmeijer J, Porte RJ, Lisman T. Hypercoagulability following major partial liver resection - detected by thrombomodulin-modified thrombin generation testing. Aliment Pharmacol Ther. 2015;41(2):189-98. 26. Groeneveld DJ, Alkozai EM, Adelmeijer J, Porte RJ, Lisman T. Balance between von Willebrand factor and ADAMTS13 following major partial hepatectomy. BJS (British Journal of Surgery). 2016;103(6):735-43.

Figure legends

Figure 1: Relationship between INR and TEG parameters on POD1 and POD3

Figure 2: Relationship between INR and factor levels on POD1 and POD3

Table 1: Patient characteristics $(n=41)$

This article is protected by copyright. All rights reserved. 


\begin{tabular}{|c|c|}
\hline Age, years - Median (range) & $61(49-73.5)$ \\
\hline \multicolumn{2}{|l|}{ Sex } \\
\hline Male & $23(56.1 \%)$ \\
\hline Female & $18(43.9 \%)$ \\
\hline Body mass index - Median (range) & $24.5(22.8-28.2)$ \\
\hline \multicolumn{2}{|l|}{ ASA } \\
\hline I & $5(12.1 \%)$ \\
\hline II & $24(58.5 \%)$ \\
\hline III & $12(29.3 \%)$ \\
\hline \multicolumn{2}{|l|}{ Pathology } \\
\hline Colorectal cancer metastases & $29(70.7 \%)$ \\
\hline Hepatocellular carcinoma & $2(4.8 \%)$ \\
\hline Neuroendocrine tumour metastases & $2(4.8 \%)$ \\
\hline Nasopharyngeal carcinoma metastases & $1(2.4 \%)$ \\
\hline Gastrointestinal stromal tumour metastases & $1(2.4 \%)$ \\
\hline Testicular neoplasm metastases & $1(2.4 \%)$ \\
\hline Undifferentiated malignancy & $1(2.4 \%)$ \\
\hline No cancer identified & $4(9.7 \%)$ \\
\hline \multicolumn{2}{|l|}{ Surgical approach } \\
\hline Open & $33(80.4 \%)$ \\
\hline Laparoscopic & $8(19.5 \%)$ \\
\hline \multicolumn{2}{|l|}{ Extent of resection } \\
\hline Major & $14(34.1 \%)$ \\
\hline Minor & $27(65.9 \%)$ \\
\hline Operative time - mins, median (range) & $200(155-272)$ \\
\hline Estimated blood loss, mL - median (range) & $150(100-250)$ \\
\hline \multicolumn{2}{|l|}{ IV fluids administered - mL, median (range) } \\
\hline Intra operative & $1500(1000-2075)$ \\
\hline POD 1 & $2000(1229-3043)$ \\
\hline POD 3 & $1540(0-2300)$ \\
\hline \multicolumn{2}{|l|}{ Haemoglobin, g/L - median (range) } \\
\hline Induction of anaesthesia & $125(119.5-135)$ \\
\hline End of surgery & $124(113-129.3)$ \\
\hline POD 1 & $117(104.5-123.3)$ \\
\hline POD 3 & $111(94-117)$ \\
\hline \multicolumn{2}{|l|}{ Haematocrit (Reference range: $0.4-0.54$ ) } \\
\hline Induction of anaesthesia & $0.37(0.35-0.4)$ \\
\hline End of surgery & $0.37(0.34-0.38)$ \\
\hline POD 1 & $0.34(0.31-0.38)$ \\
\hline POD 3 & $0.33(0.28-0.35)$ \\
\hline \multicolumn{2}{|l|}{ Mechanical thrombo-prophylaxis } \\
\hline Intra operative & $38(92.7 \%)$ \\
\hline POD 1 & $21(51.2 \%)$ \\
\hline
\end{tabular}

This article is protected by copyright. All rights reserved. 


\begin{tabular}{|l|l|}
\hline POD 3 & $17(41.4 \%)$ \\
\hline Pharmacological thrombo-prophylaxis & $2(4.9 \%)$ \\
Intra operative & $22(53.6 \%)$ \\
POD 0 & $38(92.7 \%)$ \\
POD 1 & $41(100 \%)$ \\
POD 3 & \\
\hline
\end{tabular}

\section{Supporting Information}

1. Figure S1: Temporal change in haemoglobin and haematocrit levels

2. Figure S2: Temporal change in INR, APTT, fibrinogen and d-Dimmer

3. Figure S3: Temporal change in factor levels, PPL and vWFAg

4. Figure S4: Temporal change in TEG parameters

5. Figure S5: Relationship between INR and fibrinogen, PPL and vWFAg on POD 1 and POD 3 

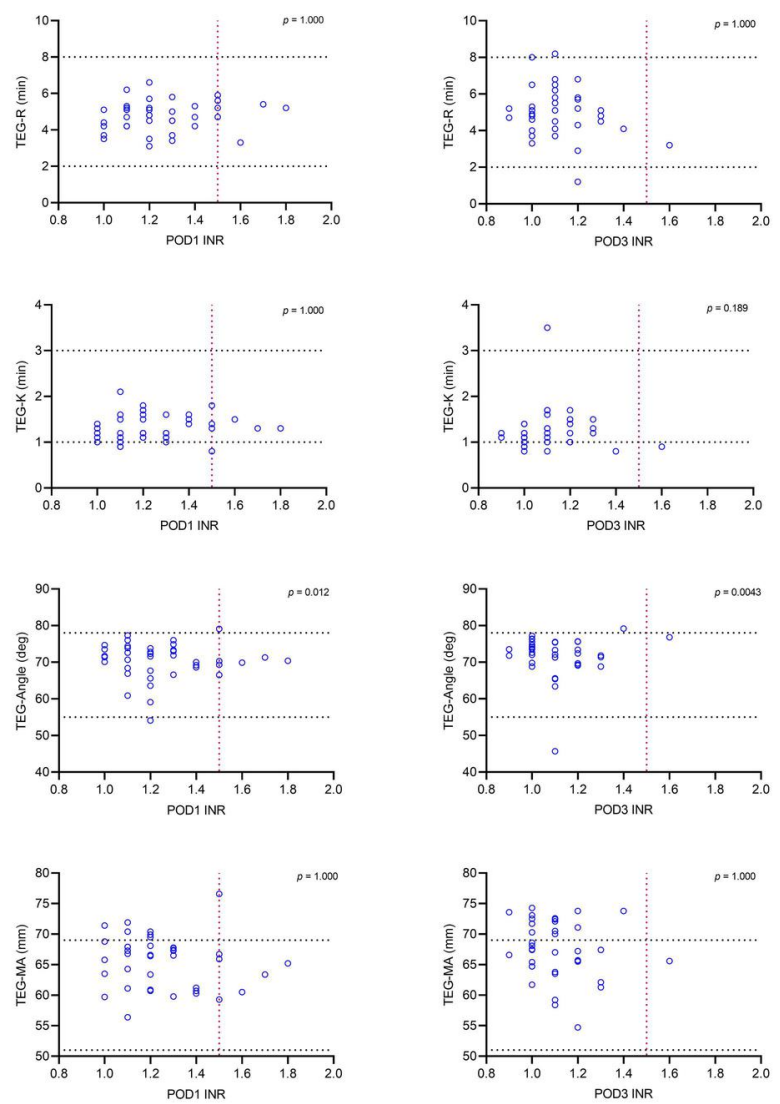

ANS_15912_Figure 1 Relationship between INR and TEG parameters post operatively.jpg

This article is protected by copyright. All rights reserved. 

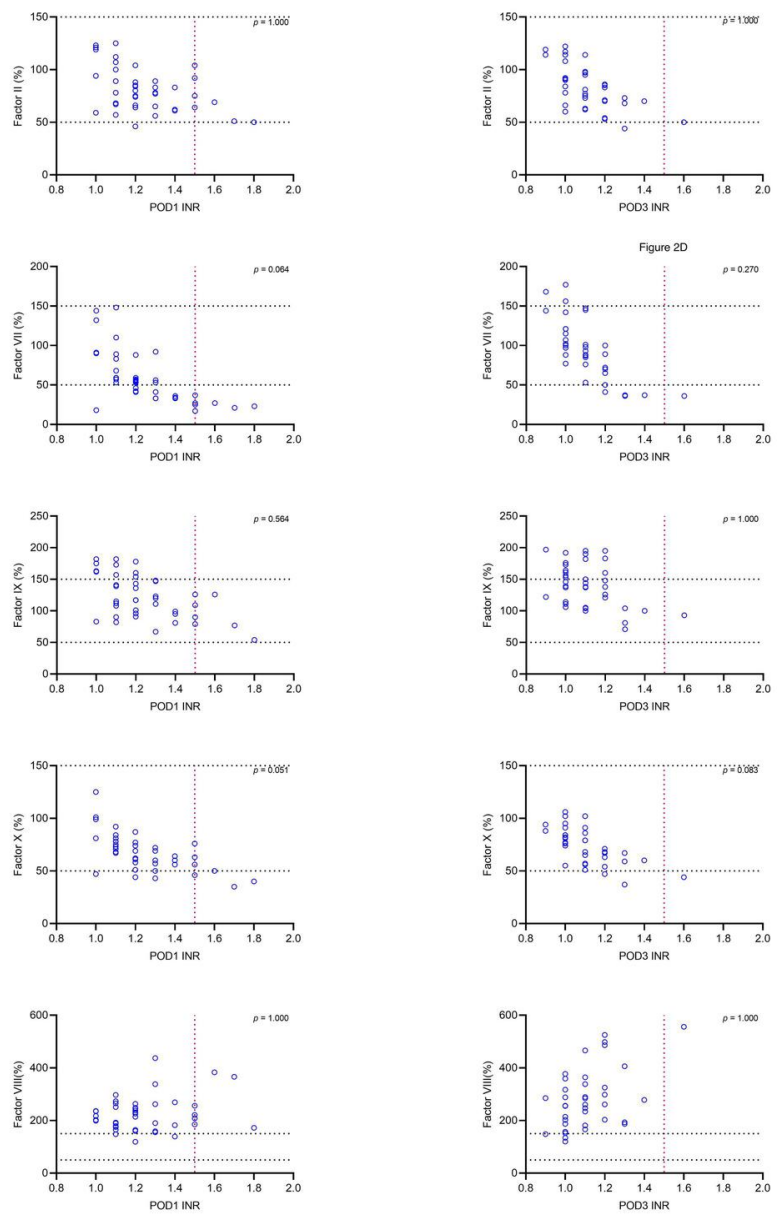

ANS_15912_Figure 2 INR vs factor levels.jpg 


\section{University Library}

\section{- M M N E R VA A gateway to Melbourne's research publications}

Minerva Access is the Institutional Repository of The University of Melbourne

\section{Author/s:}

Oo, J;Allen, M;Loveday, BPT;Lee, N;Knowles, B;Riedel, B;Burbury, K;Thomson, B

Title:

Coagulation in liver surgery: an observational haemostatic profile and thromboelastography study

\section{Date:}

2020-05-26

\section{Citation:}

Oo, J., Allen, M., Loveday, B. P. T., Lee, N., Knowles, B., Riedel, B., Burbury, K. \& Thomson, B. (2020). Coagulation in liver surgery: an observational haemostatic profile and thromboelastography study. ANZ JOURNAL OF SURGERY, 90 (6), pp.1112-1118. https:// doi.org/10.1111/ans. 15912.

Persistent Link:

http://hdl.handle.net/11343/275784 